\title{
An Approximation Method for Solving Volterra Integrodifferential Equations with a Weighted Integral Condition
}

\author{
A. Guezane-Lakoud, N. Bendjazia, and R. Khaldi \\ Laboratory of Advanced Materials, Department of Mathematics, Faculty of Sciences, Badji Mokhtar-Annaba University, \\ P.O. Box 12, 23000 Annaba, Algeria
}

Correspondence should be addressed to A. Guezane-Lakoud; a_guezane@yahoo.fr

Received 19 February 2015; Revised 23 April 2015; Accepted 23 April 2015

Academic Editor: Richard I. Avery

Copyright (C) 2015 A. Guezane-Lakoud et al. This is an open access article distributed under the Creative Commons Attribution License, which permits unrestricted use, distribution, and reproduction in any medium, provided the original work is properly cited.

We apply the reproducing kernel Hilbert space (RKHS) method for getting analytical and approximate solutions for second-order hyperbolic integrodifferential equations with a weighted integral condition. The analytical solution is represented in the form of series; thus, the $n$-terms approximate solutions are obtained. The results of the numerical examples are compared with the exact solutions to illustrate the accuracy and the effectivity of this method.

\section{Introduction}

In functional analysis, a reproducing kernel Hilbert space is a Hilbert space of functions in which pointwise evaluation is a continuous linear functional. The subject was originally developed by Aronszajn in 1950 (see [1]).

Reproducing kernel theory has important applications in numerical analysis, differential equations, integral equations, probability, and statistics [2-4]. Recently, using the RKHS method, the authors in [5-21] have discussed singular linear two-point boundary value problems, singular nonlinear twopoint periodic boundary value problems, nonlinear system of boundary value problems, initial value problems, singular integral equations, nonlinear partial differential equations, operator equations, and fourth-order integrodifferential equations (IDEs), differential equations with integral condition.

The purpose of this paper is to extend the application of the reproducing kernel Hilbert space method to solve a second-order boundary value problem of IDEs of the following Volterra type:

$$
\frac{\partial^{2} U}{\partial t^{2}}-\frac{\partial^{2} U}{\partial x^{2}}=f(x, t)+\int_{0}^{t} a(t-s) K(s, U(x, s)) d s,
$$

for all $(x, t) \in Q=(0,1) \times(0,1)$, subject to the initial conditions

$$
\begin{aligned}
& U(x, 0)=\psi_{1}(x), \\
& U_{t}(x, 0)=\psi_{2}(x), \\
& U_{x}(0, t)=\psi_{3}(t)
\end{aligned}
$$

and the weighted integral condition

$$
\int_{0}^{1} x U(x, t) d x=0
$$

where $f, \psi_{1}, \psi_{2}, \psi_{3}, a$, and $K$ are given functions, such that

$$
\begin{aligned}
& \psi_{1}^{\prime}(0)=\psi_{2}^{\prime}(0)=0, \\
& \psi_{3}(0)=\psi_{3}^{\prime}(0)=0 .
\end{aligned}
$$

The operator $K(t, u)$ is linear with respect to $u$ and continuous according to both variables $t$ and $u$. The function $a$ is continuous and denote $l=\max _{0 \leq t \leq 1}|a(t)|$.

In order to put initial conditions and integral boundary conditions of (1) into the reproducing kernel space $H(Q)$ constructed in the following sections, we have to homogenize 
these conditions. For this, let $u(x, t)=U(x, t)-\psi_{1}(x)-$ $t \psi_{2}(x)-(x-2 / 3) \psi_{3}(t)$; then the problem can be converted into the following form:

$$
\frac{\partial^{2} u}{\partial t^{2}}-\frac{\partial^{2} u}{\partial x^{2}}=F(x, t)+\int_{0}^{t} a(t-s) K(s, u(x, s)) d s
$$

for all $(x, t) \in Q$, subject to the initial conditions

$$
\begin{aligned}
& u(x, 0)=0, \\
& u_{t}(x, 0)=0, \\
& u_{x}(0, t)=0,
\end{aligned}
$$

and the weighted integral condition

$$
\int_{0}^{1} x u(x, t) d x=0
$$

The theoretical aspects of (1) with conditions (2)-(4) have been studied by Galerkin method in [22]; however, no numerical method was presented. In fact, by this procedure, the authors have found several difficulties to find the discretization of this problem due to the presence of the integral condition. Therefore, to overcome these difficulties, in the present work, we propose the RKHS method which is a simple and effective method for obtaining the numerical solution that converges rapidly to the exact solution with a small error and with a good precision. Moreover, the partial derivatives of $u_{n}(x, t)$ are also convergent to the partial of $u(x, t)$. So, from this work, we show the applicability of the RKHS method to solve this kind of problem without discretization such as Galerkin and Rothe's method. Furthermore, the RKHS method has an advantage that it is the possibility to pick any point in the domain of integration as well as in the approximate solutions and all its partial derivatives up to order two that will be applicable.

In recent years, a growing interest has been devoted to the study of IDEs which are a combination of differential and Volterra-Fredholm integral equations. IDEs appear in various fields of science such as physics, biology, and engineering. We should mention also that integrodifferential equations are usually difficult to solve analytically.

Recently, various kinds of numerical methods have been used for efficient approximation solution. In [23], authors have presented a practical matrix method for solving nonlinear Volterra-Fredholm integrodifferential equations. In [24], authors have given a numerical solution of nonlinear Volterra-Fredholm integrodifferential equations using the spectral homotopy analysis method. In [25-28], authors have obtained the representation of the exact solution for the nonlinear Volterra-Fredholm integral equations by using the reproducing kernel method.

To sum up, in this paper, we define several reproducing kernel spaces in Section 2, whereas, in Section 3, we introduce a linear operator, a complete normal orthogonal system, and some essential results. In Section 4, we provide the main results and the exact and approximate solution of (1)-(4) and we also develop an iterative method for this kind of problems.
Finally, in Section 5, the results of some numerical examples are presented and are compared with the analytical solution to confirm the good accuracy of the presented method.

\section{Reproducing Kernel Spaces}

In this section, we define some useful reproducing kernel spaces.

Definition 1. Let

$H_{3}[0,1]=\left\{u(x) \mid u, u^{\prime}\right.$,

$u^{\prime \prime}$ are absolutely continuous real value functions in $[0,1]$,

$$
\left.u^{(3)}(x) \in L^{2}[0,1], u^{\prime}(0)=\int_{0}^{1} x u(x) d x=0\right\} .
$$

Define, respectively, the inner product and the norm in $H_{3}[0,1]$ by

$$
\begin{aligned}
&\langle u(x), v(x)\rangle_{H_{3}}= \sum_{i=0}^{2} u^{(i)}(0) v^{(i)}(0) \\
&+\int_{0}^{1} u^{(3)}(x) v^{(3)}(x) d x, \\
&\|u\|_{H_{3}}= \sqrt{\langle u(x), u(x)\rangle_{H_{3}}}, \\
& u, v \in H_{3}[0,1] .
\end{aligned}
$$

We have the following result.

Theorem 2. The space $\mathrm{H}_{3}[0,1]$ is a complete reproducing kernel space; that is, for each fixed $x \in[0,1]$, there exists $\widehat{G}_{3}(y, x) \in H_{3}[0,1]$ (simply $\widehat{G}_{x}(y)$ ) and $y \in[0,1]$ such that $\left\langle u(y), \widehat{G}_{x}(y)\right\rangle_{H_{3}}=u(x)$ for any $u(y) \in H_{3}[0,1]$. The reproducing kernel $\widehat{G}_{x}(y)$ is given by

$$
\widehat{G}_{x}(y)= \begin{cases}\sum_{i=1}^{6} c_{i}(x) y^{i-1}-\frac{y^{7} C(x)}{5040}, & y \leq x, \\ \sum_{i=1}^{6} d_{i}(x) y^{i-1}-\frac{y^{7} C(x)}{5040}, & x<y,\end{cases}
$$

where

$$
\begin{aligned}
c_{1}(x)= & \frac{655}{9727}-\frac{1134}{9727 x^{2}}-\frac{378}{9727 x^{3}}+\frac{252}{9727 x^{4}} \\
& +\frac{131}{233448 x^{5}}+\frac{18}{48635 x^{7}}, \\
c_{2}(x)= & -\frac{x^{4}}{24}, \\
c_{3}(x)= & -\frac{1134}{9727}+\frac{2290}{9727 x^{2}}+\frac{2290}{29181 x^{3}}+\frac{63}{19454 x^{4}} \\
& -\frac{189}{194540 x^{5}}+\frac{9}{194540 x^{7}},
\end{aligned}
$$




$$
\begin{aligned}
& c_{4}(x)=-\frac{378}{9727}-\frac{189}{38908 x^{2}}-\frac{63}{38908 x^{3}}+\frac{21}{19454 x^{4}} \\
& -\frac{63}{194540 x^{5}}+\frac{3}{194540 x^{7}} \\
& c_{5}(x)=2520+315 x^{2}+105 x^{3}-70 x^{4}+21 x^{5} \\
& -\frac{x^{7}}{97270} \\
& c_{6}(x)=-\frac{378}{9727}-\frac{189}{194540 x^{2}}-\frac{63}{194540 x^{3}}+\frac{21}{97270 x^{4}} \\
& -\frac{63}{972700 x^{5}}+\frac{3}{972700 x^{7}} \text {, } \\
& d_{1}(x)=\frac{655}{9727}-\frac{1134}{9727 x^{2}}-\frac{378}{9727 x^{3}}+\frac{252}{9727 x^{4}} \\
& +\frac{131}{233448 x^{5}}+\frac{18}{48635 x^{7}}, \\
& d_{2}(x)=0 \text {, } \\
& d_{3}(x)=-\frac{1134}{9727}+\frac{2290}{9727 x^{2}}+\frac{2290}{29181 x^{3}}+\frac{63}{19454 x^{4}} \\
& -\frac{189}{194540 x^{5}}+\frac{9}{194540 x^{7}} \text {, } \\
& d_{4}(x)=-\frac{378}{9727}-\frac{189}{38908 x^{2}}-\frac{63}{38908 x^{3}}+\frac{21}{19454 x^{4}} \\
& -\frac{63}{194540 x^{5}}+\frac{3}{194540 x^{7}}, \\
& d_{5}(x)=\frac{252}{9727}-\frac{x}{24}+\frac{63}{19454 x^{2}}+\frac{21}{19454 x^{3}} \\
& -\frac{7}{9727 x^{4}}+\frac{21}{97270 x^{5}}-\frac{1}{97270 x^{7}} \text {, } \\
& d_{6}(x)=\frac{131}{233448}-\frac{189}{194540 x^{2}}-\frac{63}{194540 x^{3}} \\
& +\frac{21}{97270 x^{4}}-\frac{63}{972700 x^{5}}+\frac{3}{972700 x^{7}}, \\
& C(x)=-\frac{18144}{9727}-\frac{2268}{9727 x^{2}}-\frac{756}{9727 x^{3}}+\frac{504}{9727 x^{4}} \\
& -\frac{756}{48635 x^{5}}+\frac{36}{48635 x^{7}}
\end{aligned}
$$

Proof. Through several integrations by parts of (10), we obtain

$$
\begin{aligned}
& \left\langle u(y), \widehat{G}_{x}(y)\right\rangle_{H_{3}} \\
& =\sum_{i=0}^{2} u^{(i)}(0)\left[\partial_{y}^{i} \widehat{G}_{x}(0)-(-1)^{2-i} \partial_{y}^{5-i} \widehat{G}_{x}(0)\right] \\
& \quad+\sum_{i=0}^{2}(-1)^{2-i} u^{(i)}(1) \partial_{y}^{5-i} \widehat{G}_{x}(1) \\
& -\int_{0}^{1} u(y) \partial_{y}^{6} \widehat{G}_{x}(y) d y .
\end{aligned}
$$

Since $u(x) \in H_{3}[0,1]$, we have

$$
u^{\prime}(0)=\int_{0}^{1} x u(x) d x=0
$$

Then

$$
\begin{aligned}
\left\langle u(y), \widehat{G}_{x}(y)\right\rangle_{H_{3}}= & u(0)\left[\widehat{G}_{x}(0)-\partial_{y}^{5} \widehat{G}_{x}(0)\right] \\
& +u^{(2)}(0)\left[\partial_{y}^{2} \widehat{G}_{x}(0)-\partial_{y}^{3} \widehat{G}_{x}(0)\right] \\
& +\sum_{i=0}^{2}(-1)^{2-i} u^{(i)}(1) \partial_{y}^{5-i} \widehat{G}_{x}(1) \\
& -\int_{0}^{1} u(y) \partial_{y}^{6} \widehat{G}_{x}(y) d y \\
& -C(x) \int_{0}^{1} y u(y) d y .
\end{aligned}
$$

Note that the property of the reproducing kernel $\left\langle u(y), \widehat{G}_{x}(y)\right\rangle_{H_{3}}=u(x)$; then $\widehat{G}_{x}(y)$ is the solution of the following generalized differential equation:

$$
\partial_{y}^{6} \widehat{G}_{x}(y)+C(x) y=-\delta(y-x)
$$

with the boundary conditions

$$
\begin{aligned}
\widehat{G}_{x}(0)-\partial_{y}^{5} \widehat{G}_{x}(0) & =0, \\
\partial_{y}^{2} \widehat{G}_{x}(0)-\partial_{y}^{3} \widehat{G}_{x}(0) & =0, \\
\partial_{y}^{5-i} \widehat{G}_{x}(1) & =0, \quad i=0,1,2 .
\end{aligned}
$$

As $x \neq y$, it follows that

$$
\widehat{G}_{x}(y)= \begin{cases}\sum_{i=1}^{6} c_{i}(x) y^{i-1}-\frac{y^{7} C(x)}{5040}, & y \leq x, \\ \sum_{i=1}^{6} d_{i}(x) y^{i-1}-\frac{y^{7} C(x)}{5040}, & x<y .\end{cases}
$$

Let us find the coefficients $c_{i}(x), d_{i}(x)$, and $C(x)$. Since $\partial_{y}^{6} \widehat{G}_{x}(y)+C(x) y=-\delta(y-x)$, we have

$$
\begin{aligned}
\partial_{y}^{5} \widehat{G}_{x}(x-0)-\partial_{y}^{5} \widehat{G}_{x}(x+0) & =1, \\
\partial_{y}^{i} \widehat{G}_{x}(x+0) & =\partial_{y}^{i} \widehat{G}_{x}(x-0),
\end{aligned}
$$


Since $\widehat{G}_{x}(y) \in H_{3}[0,1]$, it follows that

$$
\begin{array}{r}
\partial_{y}^{1} \widehat{G}_{x}(0)=0, \\
\int_{0}^{1} y \widehat{G}_{x}(y) d y=0 .
\end{array}
$$

Through (17)-(20), the unknown coefficients of (11) can be obtained.

Definition 3. Define the space

$$
\begin{aligned}
& H_{3}^{\prime}[0,1] \\
& =\left\{\begin{array}{c}
u(t) \mid u, u^{\prime}, u^{\prime \prime} \text { are absolutely continuous functions in }[0,1] \\
u^{(3)}(t) \in L^{2}[0,1], u^{\prime}(0)=u(0)=0
\end{array}\right\} .
\end{aligned}
$$

$$
H(Q)=\left\{\begin{array}{c}
u \mid \frac{\partial^{4} u}{\partial^{2} x \partial^{2} t} \text { is absolutely continuous real-valued function in } Q, \\
\frac{\partial^{6} u}{\partial^{3} x \partial^{3} t} \in L^{2}(Q), u(x, 0)=\frac{\partial u(x, 0)}{\partial t}=0, \frac{\partial u(0, t)}{\partial x}=\int_{0}^{1} x u(x, t) d x=0
\end{array}\right\}
$$

The inner product and the norm in $H(Q)$ are defined, respectively, by

$$
\begin{aligned}
& \langle u(x, t), v(x, t)\rangle_{H} \\
& =\sum_{i=0}^{2} \int_{0}^{1}\left[\frac{\partial^{3}}{\partial t^{3}} \frac{\partial^{i}}{\partial t^{i}} u(0, t) \frac{\partial^{3}}{\partial t^{3}} \frac{\partial^{i}}{\partial t^{i}} v(0, t)\right] d t \\
& +\sum_{j=0}^{2}\left\langle\frac{\partial^{j}}{\partial t^{j}} u(x, 0), \frac{\partial^{j}}{\partial t^{j}} v(x, 0)\right\rangle_{H_{3}} \\
& +\int_{0}^{1} \int_{0}^{1}\left[\frac{\partial^{6}}{\partial x^{3} \partial t^{3}} u(x, t) \frac{\partial^{6}}{\partial x^{3} \partial t^{3}} v(x, t)\right] d t d x \\
& \|u\|_{H_{(Q)}}=\sqrt{\langle u(x, t), u(x, t)\rangle_{H}} \cdot
\end{aligned}
$$

$H(Q)$ is a reproducing kernel space and its reproducing kernel function is

$$
G_{(y, s)}(x, t)=\widehat{G}_{x}(y) \breve{G}_{s}(t)
$$

such that, for any $u(x, t) \in H(Q)$,

$$
\begin{aligned}
u(y, s) & =\left\langle u(x, t), G_{(y, s)}(x, t)\right\rangle_{H}, \\
G_{(x, t)}(y, s) & =G_{(y, s)}(x, t) .
\end{aligned}
$$

The inner product and the norm in $H_{3}^{\prime}[0,1]$ are defined, respectively, by

$$
\begin{aligned}
\langle u(t), v(t)\rangle_{H_{3}^{\prime}}= & \sum_{i=0}^{2} u^{(i)}(0) v^{(i)}(0) \\
& +\int_{0}^{1} u^{(3)}(x) v^{(3)}(x) d x \\
\|u\|_{H_{3}^{\prime}}= & \sqrt{\langle u(t), u(t)\rangle_{H_{3}^{\prime}}}
\end{aligned}
$$

The space $H_{3}^{\prime}[0,1]$ is a complete reproducing kernel space, and its reproducing kernel $\breve{G}_{s}(t)$ is given by

$$
\breve{G}_{s}(t)= \begin{cases}\frac{1}{120} s^{5}-\frac{1}{24} s^{4} t+\frac{1}{4} s^{2} t^{2}+\frac{1}{12} s^{3} t^{2}, & s \leq t, \\ -\frac{1}{24} s^{4} t+\frac{1}{120} t^{5}+\frac{1}{12} s^{2} t^{2}(3+t), & t<s .\end{cases}
$$

Definition 4. Define 
The inner product and the norm in $\widehat{H}(Q)$ are defined, respectively, by

$$
\begin{aligned}
& \langle u(x, t), v(x, t)\rangle_{\widehat{H}} \\
& =\int_{0}^{1}\left[\frac{\partial}{\partial t} u(0, t) \frac{\partial}{\partial t} v(0, t)\right] d t \\
& \quad+\langle u(x, 0), v(x, 0)\rangle_{H_{1}} \\
& \quad+\int_{0}^{1} \int_{0}^{1}\left[\frac{\partial^{2}}{\partial x \partial t} u(x, t) \frac{\partial^{2}}{\partial x \partial t} v(x, t)\right] d t d x, \\
& \|u\|_{\widehat{H}}=\sqrt{\langle u(x, t), u(x, t)\rangle_{\widehat{H}}} .
\end{aligned}
$$

$\widehat{H}(Q)$ is a complete reproducing kernel space; thus, its reproducing kernel function is (see [11])

$$
R_{(y, s)}(x, t)=\dot{R}_{y}(x) \dot{R}_{s}(t),
$$

where

$$
\dot{R}_{y}(x)= \begin{cases}1+y, & y \leq x \\ 1+x, & x<y\end{cases}
$$

\section{A Bounded Linear Operator on $H(Q)$}

Define differential operator $L: H(Q) \rightarrow \widehat{H}(Q)$ by

$$
L u(x, t)=\frac{\partial^{2} u}{\partial t^{2}}-\frac{\partial^{2} u}{\partial x^{2}}
$$

therefore, (1) can be converted into an equivalent form as follows:

$$
\operatorname{Lu}(x, t)=\widetilde{F}(x, t, u(x, t)),
$$

where $u(x, t) \in H(Q), \widetilde{F}(x, t, u(x, t)) \in \widehat{H}(Q)$, and

$$
\begin{aligned}
\widetilde{F}(x, t, u(x, t))= & f(x, t) \\
& +\int_{0}^{t} a(t-s) K(s, u(x, s)) d s .
\end{aligned}
$$

It is easy to show that $L$ is a bounded linear operator from $H(Q)$ to $\widehat{H}(Q)$. Now, we choose a countable subset $S=$ $\left\{\left(x_{1}, t_{1}\right),\left(x_{2}, t_{2}\right), \ldots\right\}$ in $Q$ and we define

$$
\begin{aligned}
& \varphi_{i}(x, t)=R_{\left(x_{i}, t_{i}\right)}(x, t), \\
& \omega_{i}(x, t)=L^{*} \varphi_{i}(x, t) .
\end{aligned}
$$

Lemma 7. Assume that $S$ is dense in $Q$; then, $\left\{\omega_{i}(x, t)\right\}_{i=1}^{\infty}$ is a complete system in $H(Q)$, and

$$
\omega_{i}(x, t)=\left(L_{(y, s)} G_{(y, s)}(x, t)\right), \quad \text { for }(y, s)=\left(x_{i}, t_{i}\right) .
$$

Applying Gram-Schmidt process, we obtain an orthogonal basis $\left\{\omega_{i}(x, t)\right\}_{i=1}^{\infty}$ of $H(Q)$, such that

$$
\omega_{i}(x, t)=\sum_{j=1}^{i} \beta_{i j} \omega_{i}(x, t),
$$

where $\beta_{i j}$ are orthogonal coefficients.

\section{The Exact and Approximate Solution}

Lemma 8. If $S$ is dense in $Q$, then the exact solution of (35) is

$$
u(x, t)=\sum_{i=1}^{\infty} \sum_{j=1}^{i} \beta_{i j} \widetilde{F}\left(x_{j}, t_{j}, u\left(x_{j}, t_{j}\right)\right) \omega_{i}(x, t)
$$

Proof. The exact solution $u(x, t)$ can be expanded in the Fourier series in terms of normal orthogonal basis $\left\{\omega_{i}(x, t)\right\}_{i=1}^{\infty}$ in $H(Q)$ :

$$
\begin{aligned}
u(x, t) & \\
= & \sum_{i=1}^{\infty}\left\langle u(x, t), \omega_{i}(x, t)\right\rangle_{H} \omega_{i}(x, t) \\
& =\sum_{i=1}^{\infty} \sum_{j=1}^{i} \beta_{i j}\left\langle u(x, t), \omega_{j}(x, t)\right\rangle_{H} \omega_{i}(x, t) \\
& =\sum_{i=1}^{\infty} \sum_{j=1}^{i} \beta_{i j}\left\langle u(x, t), L^{*} \varphi_{i}(x, t)\right\rangle_{H} \omega_{i}(x, t) \\
& =\sum_{i=1}^{\infty} \sum_{j=1}^{i} \beta_{i j}\left\langle L u(x, t), \varphi_{i}(x, t)\right\rangle_{\widehat{H}(\mathrm{Q})} \omega_{i}(x, t) \\
& =\sum_{i=1}^{\infty} \sum_{j=1}^{i} \beta_{i j}\left\langle L u(x, t), R_{\left(x_{i}, t_{i}\right)}(x, t)\right\rangle_{\widehat{H}(\mathrm{Q})} \omega_{i}(x, t) \\
& =\sum_{i=1}^{\infty} \sum_{j=1}^{i} \beta_{i j}(L u)\left(x j, t_{j}\right) \omega_{i}(x, t) \\
& =\sum_{i=1}^{\infty} \sum_{j=1}^{i} \beta_{i j} \widetilde{F}\left(x, t_{j}, u\left(x_{j}, t_{j}\right)\right) \omega_{i}(x, t) .
\end{aligned}
$$

4.1. Iterative Method. We construct an iterative method to obtain the approximate solutions of (1) in the reproducing kernel space $H(Q)$. Let

$$
A_{i}=\sum_{j=1}^{i} \beta_{i j} \widetilde{F}\left(x_{j}, t_{j}, u\left(x_{j}, t_{j}\right)\right) .
$$

Then

$$
u(x, t)=\sum_{i=1}^{\infty} A_{i} \omega_{i}(x, t)
$$

Define the initial function $u_{0}(x, t)=0$ and the $n$-term approximation to $u(x, t)$ by

$$
u_{n}(x, t)=\sum_{i=1}^{n} \widetilde{A}_{i} \omega_{i}(x, t)
$$


where the coefficients $\widetilde{A}_{i}$ are given as

$$
\begin{gathered}
\widetilde{A}_{1}=\beta_{11} \widetilde{F}\left(x_{1}, t_{1}, u_{0}\left(x_{1}, t_{1}\right)\right), \\
\widetilde{A}_{2}=\sum_{j=1}^{2} \beta_{2 j} \widetilde{F}\left(x_{j}, t_{j}, u_{j-1}\left(x_{j}, t_{j}\right)\right), \\
\vdots \\
\widetilde{A}_{n}=\sum_{j=1}^{n} \beta_{n j} \widetilde{F}\left(x_{j}, t_{j}, u_{j-1}\left(x_{j}, t_{j}\right)\right) .
\end{gathered}
$$

Theorem 9. If $S$ is dense in $Q$, then

$$
\left(L u_{n}\right)(x, t)=\widetilde{F}\left(x, t, u_{n-1}(x, t)\right),
$$

where $u_{n}(x, t)$ is given by $(44)$.

Theorem 10. Suppose that $\left\|\int_{0}^{t} K(s, u(x, s)) d s\right\|_{\widehat{H}(\mathrm{Q})} \leq$ $C\|u\|_{H(Q)}$ and $0<l C\left\|L^{-1}\right\|<1$; then

$$
u_{n}(x, t) \stackrel{\|\cdot\|_{H}}{\longrightarrow} u(x, t) \quad \text { as }(n \longrightarrow \infty) .
$$

Proof. From the preceding theorem, we have

$$
\left(L u_{n}\right)(x, t)=\widetilde{F}\left(x, t, u_{n-1}(x, t)\right)
$$

so

$$
\begin{gathered}
\left\|u_{n}(x, t)-u(x, t)\right\|_{H}=\| L^{-1}\left(\widetilde{F}\left(x, t, u_{n-1}(x, t)\right)\right. \\
-\widetilde{F}(x, t, u(x, t)))\left\|_{H} \leq\right\| L^{-1}\|\|\left(\widetilde{F}\left(x, t, u_{n-1}(x, t)\right)\right. \\
-\widetilde{F}(x, t, u(x, t)))\left\|_{\widehat{H}(\mathrm{Q})} \leq\right\| L^{-1}\|\|\left(\int_{0}^{t} a(t-s)\right. \\
\left.\cdot K\left(s, u_{n-1}(x, s)-u(x, s)\right) d s\right) \|_{\widehat{H}(Q)} \leq C \\
\cdot \max _{0 \leq t \leq 1}|a(t)|\left\|L^{-1}\right\|\left\|u_{n-1}(x, t)-u(x, t)\right\|_{H} .
\end{gathered}
$$

From $l C\left\|L^{-1}\right\|<1$ and (49), we get

$$
u_{n}(x, t) \stackrel{\|\cdot\|_{H}}{\longrightarrow} u(x, t) \quad \text { as } n \longrightarrow \infty .
$$

Proposition 11. Suppose that $u_{n}(x, t) \stackrel{\|\cdot\|_{H}}{\longrightarrow} u(x, t)$ as $(n \rightarrow$ $\infty)$; then

$$
\begin{aligned}
\left|\frac{\partial^{i+j}}{\partial x^{i} \partial t^{j}} u(x, t)-\frac{\partial^{i+j}}{\partial x^{i} \partial t^{j}} u_{n}(x, t)\right| & \longrightarrow 0, \\
n & \longrightarrow \infty, i, j=0,1,2 .
\end{aligned}
$$

Now, the approximate solution $u_{n}^{m}(x, t)$ can be obtained by taking finitely many terms in the series representation of $u_{n}(x, t)$ and

$$
u_{n}^{m}(x, t)=\sum_{i=1}^{m} \sum_{j=1}^{i} \beta_{i j} \widetilde{F}\left(x_{j}, t_{j}, u_{n-1}\left(x_{j}, t_{j}\right)\right) \Phi_{i}(x, t) .
$$

\section{Numerical Examples}

In order to calculate the approximate solution $u_{n}^{m}(x, t)$, we take

$$
\begin{aligned}
\left(x_{j}, t_{j}\right) & =\left(\frac{i}{N}, \frac{k}{M}\right), \\
i= & 0,1, \ldots N, k=0,1, \ldots M, j=0,1,2, \ldots \\
m & =(N+1)(M+1), \\
e_{n}^{m}(x, t) & =\left|u(x, t)-u_{n}^{m}(x, t)\right|, \\
\left(e_{n}^{m}\right)_{x x}(x, t) & =\left|u_{x x}(x, t)-\left(u_{n}^{m}\right)_{x x}(x, t)\right|, \\
\left(e_{n}^{m}\right)_{t}(x, t) & =\left|u_{t}(x, t)-\left(u_{n}^{m}\right)_{t}(x, t)\right|, \\
\left(e_{n}^{m}\right)_{x t} & =\left|u_{x t}(x, t)-\left(u_{n}^{m}\right)_{x t}(x, t)\right|, \\
\left(e_{n}^{m}\right)_{t t}(x, t) & =\left|u_{t t}(x, t)-\left(u_{n}^{m}\right)_{t t}(x, t)\right|, \\
\left(e_{n}^{m}\right)_{x}(x, t) & =\left|u_{x}(x, t)-\left(u_{n}^{m}\right)_{x}(x, t)\right| .
\end{aligned}
$$

Example 1. Consider the following hyperbolic integrodifferential equation:

$$
\frac{\partial^{2} u}{\partial t^{2}}-\frac{\partial^{2} u}{\partial x^{2}}=f(x, t)+\int_{0}^{t}(t-s)^{4} u(x, s) d s,
$$

for all $(x, t) \in Q$, subject to the initial conditions

$$
\begin{aligned}
u(x, 0) & =\frac{\partial u(x, 0)}{\partial t}=0, \\
\frac{\partial u(0, t)}{\partial x} & =t^{2}
\end{aligned}
$$

and the weighted integral condition

$$
\int_{0}^{1} x u(x, t) d x=0
$$

where

$$
f(x, t)=-4+\frac{\left(2 t^{7}\right)}{105}+\exp x\left(2-t^{2}-\frac{t^{7}}{105}\right) .
$$

The exact solution is given by $u(x, t)=(\exp x-2) t^{2}$. In order to homogenize the initial conditions, we put $v(x, t)=$ $u(x, t)-(x-2 / 3) t^{2}$; then the problem can be converted into the following form:

$$
\begin{aligned}
\frac{\partial^{2} v}{\partial t^{2}}-\frac{\partial^{2} v}{\partial x^{2}} & =F(x, t)+\int_{0}^{t}(t-s)^{4} v(x, s) d s, \\
v(x, 0) & =\frac{\partial v(x, 0)}{\partial t}=0, \\
\frac{\partial v(0, t)}{\partial x} & =0 \\
\int_{0}^{1} x v(x, t) d x & =0,
\end{aligned}
$$


TABLE 1: Comparison of results for $u(n=2, m=25,121)$.

\begin{tabular}{lccccc}
\hline$\left(x_{i}, t_{i}\right)$ & $u$ & $u_{2}^{25}$ & $e_{2}^{25}$ & $u_{2}^{121}$ & $e_{2}^{121}$ \\
\hline$(0.00,0.01)$ & -0.0000333333 & -0.0000333637 & $3.033825 \times 10^{-8}$ & -0.0000333333 & $1.27745 \times 10^{-8}$ \\
$(0.10,0.05)$ & -0.000820406 & -0.00082375 & $3.34428 \times 10^{-6}$ & -0.000820406 & $1.2258 \times 10^{-6}$ \\
$(0.15,0.10)$ & -0.00321499 & -0.00323799 & 0.000022998 & -0.00321499 & $6.31116 \times 10^{-6}$ \\
$(0.35,0.20)$ & -0.0105706 & -0.0106773 & 0.000106649 & -0.0105706 & 0.0000198708 \\
$(0.49,0.31)$ & -0.0183567 & -0.0185277 & 0.000170909 & -0.0183885 & 0.0000317253 \\
$(0.57,0.45)$ & -0.0273509 & -0.0275692 & 0.000218296 & -0.0273509 & 0.0000385202 \\
$(0.69,0.52)$ & -000800865 & -0.00804727 & 0.0000386212 & -0.00800874 & $8.8295 \times 10^{-8}$ \\
$(0.73,0.71)$ & 0.0059218 & 0.00598197 & 0.0000601728 & 0.00594941 & 0.0000276115 \\
$(0.81,0.73)$ & 0.0557278 & 0.0560604 & 0.000332616 & 0.0558007 & 0.0000728933 \\
$(0.99,0.99)$ & 0.36058 & 0.362213 & 0.00163333 & 0.360756 & 0.000176542 \\
\hline
\end{tabular}

TABLE 2: Comparison of results for $u_{x}(n=2, m=121)$.

\begin{tabular}{|c|c|c|c|c|c|c|}
\hline$\left(x_{i}, t_{i}\right)$ & $u_{x}$ & $\left(u_{2}^{121}\right)_{x}$ & $\left(e_{2}^{121}\right)_{x}$ & $u_{t}$ & $\left(u_{2}^{121}\right)_{t}$ & $\left(e_{2}^{121}\right)_{t}$ \\
\hline$(0.00,0.01)$ & 0.00000 & 0.0000 & 0.00000 & -0.00666667 & -0.00667043 & $3.76 \times 10^{-6}$ \\
\hline$(0.10,0.05)$ & 0.000262927 & 0.000263362 & $4.34808 \times 10^{-7}$ & -0.0328162 & -0.0328162 & 0.000065 \\
\hline$(0.15,0.10)$ & 0.00161834 & 0.0016219 & $3.5546 \times 10^{-6}$ & -0.0642998 & -0.0644248 & 0.000124 \\
\hline$(0.35,0.20)$ & 0.0167627 & 0.0167986 & 0.0000359083 & -0.105706 & -0.105901 & 0.000194 \\
\hline$(0.49,0.31)$ & 0.0607656 & 0.0608914 & 0.000125813 & -0.118431 & -0.118615 & 0.00018 \\
\hline$(0.57,0.45)$ & 0.155574 & 0.155866 & 0.000291519 & -0.12156 & -0.121689 & 0.00012 \\
\hline$(0.69,0.52)$ & 0.268701 & 0.269116 & 0.000415711 & -0.0308025 & -0.0307829 & 0.000019 \\
\hline$(0.73,0.71)$ & 0.541948 & 0.54252 & 0.00057215 & 0.0166811 & 0.0167237 & 0.000042 \\
\hline$(0.81,0.73)$ & 0.66501 & 0.665538 & 0.000527674 & 0.152679 & 0.152725 & 0.000045 \\
\hline$(0.99,0.99)$ & 1.65758 & 1.659 & 0.00142569 & 0.728444 & 0.727913 & 0.00053 \\
\hline
\end{tabular}

TABLE 3: Comparison of results for $u_{x x}(n=2, m=121)$.

\begin{tabular}{|c|c|c|c|c|c|c|}
\hline$\left(x_{i}, t_{i}\right)$ & $u_{x x}$ & $\left(u_{2}^{121}\right)_{x x}$ & $\left(e_{2}^{121}\right)_{x x}$ & $u_{t t}$ & $\left(u_{2}^{121}\right)_{t t}$ & $\left(e_{2}^{121}\right)_{t t}$ \\
\hline$(0.00,0.01)$ & 0.0001000 & 0.000100171 & $1.71 \times 10^{-7}$ & -0.666667 & -0.667391 & 0.000724 \\
\hline$(0.10,0.05)$ & 0.00276293 & 0.00277046 & $7.52 \times 10^{-6}$ & -0.656325 & -0.658253 & 0.001928 \\
\hline$(0.15,0.10)$ & 0.0116183 & 0.0116374 & 0.0000190 & -0.642998 & -0.64296 & 0.0000379 \\
\hline$(0.35,0.20)$ & 0.0567627 & 0.0568428 & 0.0000801 & -0.528532 & -0.528362 & 0.000169 \\
\hline$(0.49,0.31)$ & 0.156866 & 0.157201 & 0.0003349 & -0.382034 & -0.382073 & 0.000038 \\
\hline$(0.57,0.45)$ & 0.358074 & 0.35823 & 0.0001562 & -0.270133 & -0.270318 & 0.0001851 \\
\hline$(0.69,0.52)$ & 0.539101 & 0.539354 & 0.0002531 & -0.0592356 & -0.0059133 & 0.0001026 \\
\hline$(0.73,0.71)$ & 1.04605 & 1.04597 & 0.0000733 & 0.0234945 & 0.0232241 & 0.00027045 \\
\hline$(0.81,0.73)$ & 1.19791016 & 1.19798 & 0.00006967 & 0.209149 & 0.208289 & 0.00085993 \\
\hline$(0.99,0.99)$ & 2.63768 & 2.64076 & 0.003076 & 0.735802 & 0.735802 & 0.00426859 \\
\hline
\end{tabular}

where

$$
\begin{aligned}
F(x, t)= & -\frac{3}{315}\left(t^{7}+105 t^{2}-210\right) \exp x \\
& -\frac{3}{315}\left((4+3 x)+\left(-210+t^{7}\right)\right) .
\end{aligned}
$$

The numerical results are presented in Tables 1, 2, 3, and 4 and Figures 1 and 2.
Example 2. Consider the following hyperbolic integrodifferential equation:

$$
\begin{aligned}
\frac{\partial^{2} u}{\partial t^{2}}-\frac{\partial^{2} u}{\partial x^{2}} & =f(x, t)+\int_{0}^{t}\left(\int_{0}^{x} u(r, s) d r\right) d s \\
u(x, 0) & =\frac{\partial u(x, 0)}{\partial t}=0 \\
\frac{\partial u(0, t)}{\partial x} & =0 \\
\int_{0}^{1} x u(x, t) d x & =0
\end{aligned}
$$


TABLE 4: Comparison of results for $u_{x t}(n=2, m=121)$.

\begin{tabular}{lccc}
\hline$\left(x_{i}, t_{i}\right)$ & $u_{x t}$ & $\left(u_{2}^{121}\right)_{x t}$ & $\left(e_{2}^{121}\right)_{x t}$ \\
\hline$(0.00,0.01)$ & 0.00000 & 0.0000 & 0.00000 \\
$(0.10,0.05)$ & 0.0105171 & 0.0105402 & 0.0000231397 \\
$(0.15,0.10)$ & 0.0323668 & 0.0324379 & 0.0000710837 \\
$(0.35,0.20)$ & 0.167627 & 0.167991 & 0.000364067 \\
$(0.49,0.31)$ & 0.392036 & 0.392836 & 0.000799951 \\
$(0.57,0.45)$ & 0.69144 & 0.692595 & 0.00115446 \\
$(0.69,0.52)$ & 1.03346 & 1.03456 & 0.00109998 \\
$(0.73,0.71)$ & 1.52661 & 1.52694 & 0.000326263 \\
$(0.81,0.73)$ & 1.82195 & 1.82199 & 0.000041932 \\
$(0.99,0.99)$ & 3.34864 & 3.34924 & 0.000598899 \\
\hline
\end{tabular}

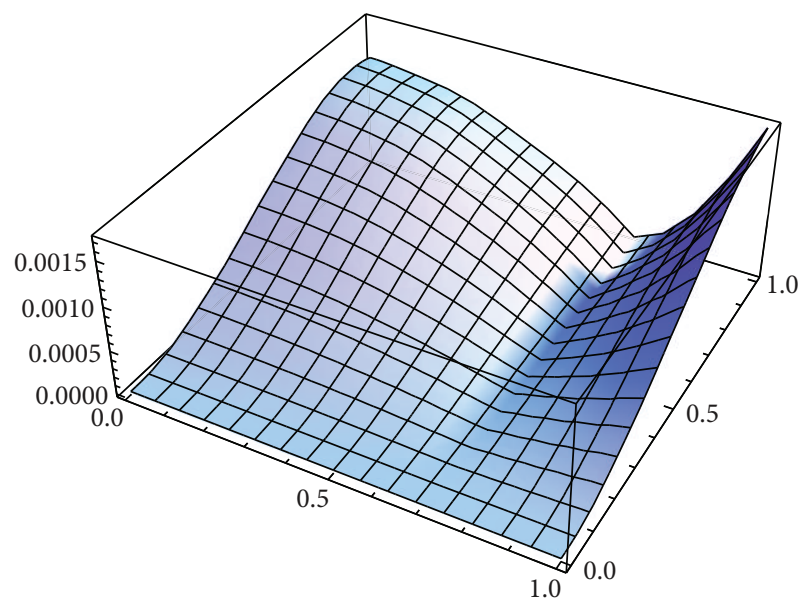

Figure 1: Consider $e_{2}^{25}(x, t)=\left|u(x, t)-u_{2}^{25}(x, t)\right|$.

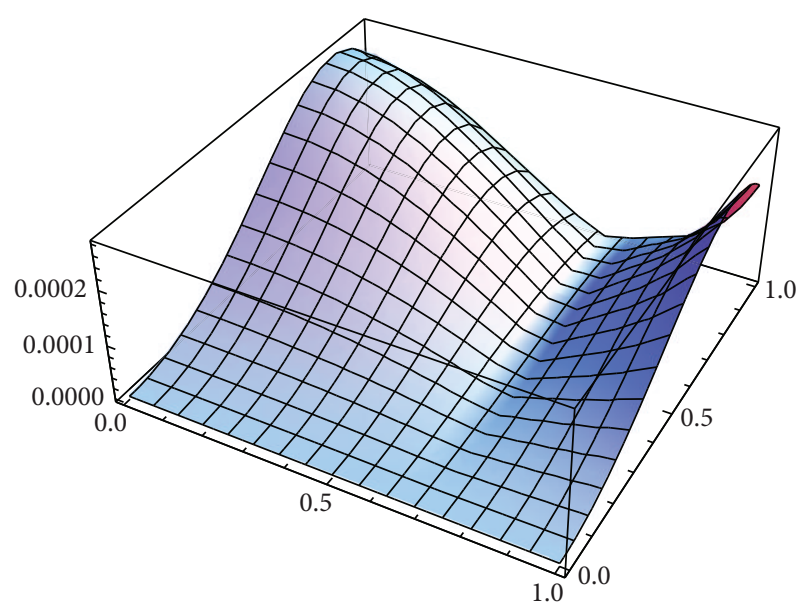

Figure 2: Consider $e_{2}^{121}(x, t)=\left|u(x, t)-u_{2}^{121}(x, t)\right|$.

where

$$
f(x, t)=-2 t^{2}+\frac{x t^{3}}{6}-\frac{x^{3} t^{3}}{9}+\left(-\left(\frac{1}{2}\right)+x^{2}\right) .
$$

The exact solution is given by $u(x, t)=\left(x^{2}-1 / 2\right) t^{2}$.

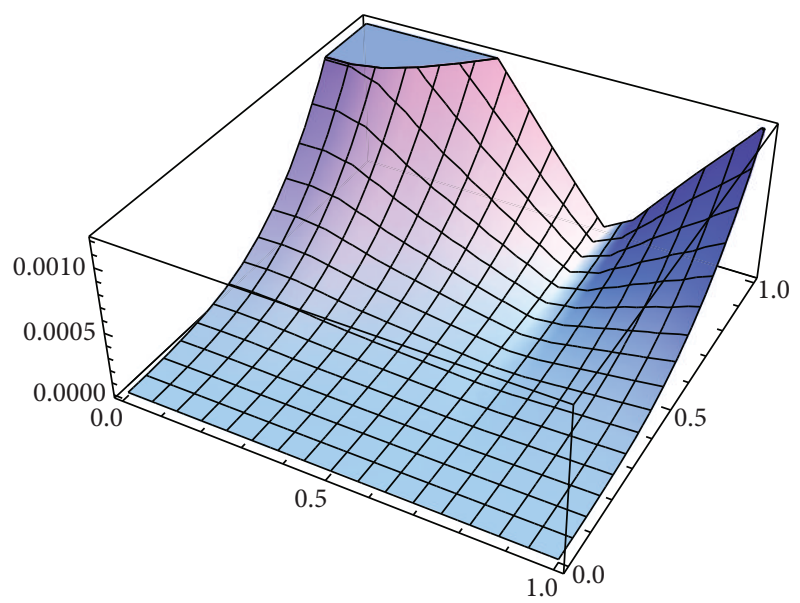

Figure 3: Consider $e_{2}^{25}(x, t)=\left|u(x, t)-u_{2}^{25}(x, t)\right|$.

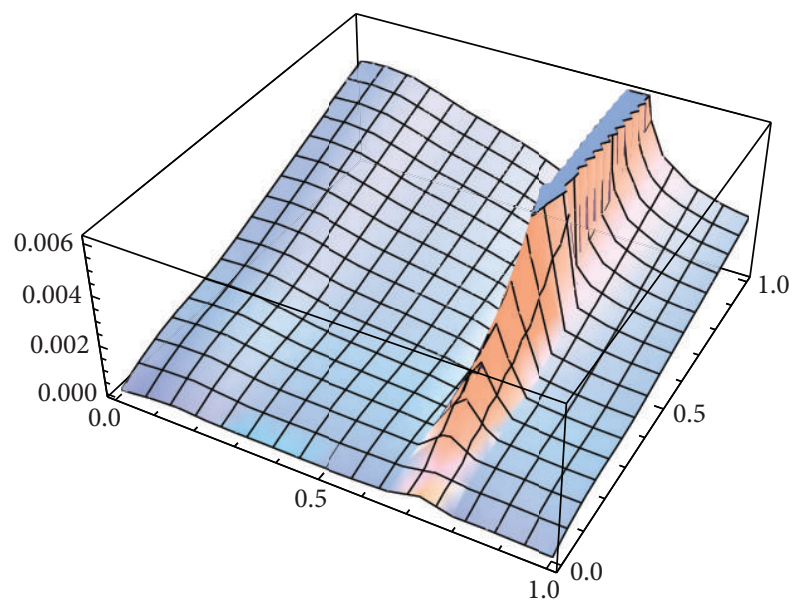

Figure 4: Consider $\left|u(x, t)-u_{2}^{25}(x, t)\right| /|u(x, t)|$.

The numerical results are presented in Tables 5, 6, 7, and 8 and Figures 3 and 4.

\section{Conclusion}

In this paper, we have applied RKHS method to solve a hyperbolic integrodifferential equation with a weighted integral condition. The numeric results have demonstrated that only a small number of iterations can be used to obtain numeric results with a good precision. From this work, we see that the absolute errors are monotonically decreasing if $m$ increase. The numerical examples show that the approximate solution and its partial derivatives of a superior order or equal to two converge to the exact solution and its partial derivatives, respectively. Consequently, we confirm that this method is effective for the integrodifferential equation with weighted integral conditions. All the computations are performed using Mathematica 9.0. 
TABLE 5: Comparison of results for $u(n=2, m=25)$.

\begin{tabular}{lcccc}
\hline$\left(x_{i}, t_{i}\right)$ & $u$ & $u_{2}^{25}$ & $e_{2}^{25}$ & $e_{2}^{25} / u$ \\
\hline$(0.00,0.01)$ & -0.000050000 & -0.0000500038 & $3.81237 \times 10^{-9}$ & 0.0000762474 \\
$(0.10,0.05)$ & -0.001225000 & -0.00122559 & $5.90641 \times 10^{-7}$ & 0.000482156 \\
$(0.15,0.10)$ & -0.004775 & -0.0047785 & $3.49902 \times 10^{-6}$ & 0.000732779 \\
$(0.35,0.20)$ & -0.01510000 & -0.0151099 & $9.9186 \times 10^{-6}$ & 0.000656861 \\
$(0.49,0.31)$ & -0.0249764 & -0.0249918 & 0.0000153856 & 0.000616005 \\
$(0.57,0.45)$ & -0.0354578 & -0.0354835 & 0.0000257761 & 0.000726954 \\
$(0.69,0.52)$ & -0.00646256 & -0.00645257 & $9.99319 \times 10^{-6}$ & 0.00154632 \\
$(0.73,0.71)$ & 0.0165849 & 0.0166669 & 0.0000819828 & 0.00494322 \\
$(0.81,0.73)$ & 0.0831857 & 0.083387 & 0.000201347 & 0.00242045 \\
$(0.99,0.99)$ & 0.470546 & 0.471707 & 0.00116079 & 0.00246689 \\
\hline
\end{tabular}

TABLE 6: Comparison of results for $u_{x}(n=2, m=25)$.

\begin{tabular}{|c|c|c|c|c|c|c|}
\hline$\left(x_{i}, t_{i}\right)$ & $u_{x}$ & $\left(u_{2}^{25}\right)_{x}$ & $\left(e_{2}^{25}\right)_{x}$ & $u_{t}$ & $\left(u_{2}^{25}\right)_{t}$ & $\left(e_{2}^{25}\right)_{t}$ \\
\hline$(0.00,0.01)$ & 0.0000 & 0.0000 & 0.000 & -0.010 & -0.0100011 & $1.13 \times 10^{-6}$ \\
\hline$(0.10,0.05)$ & 0.0005 & 0.000499228 & $7.71 \times 10^{-7}$ & -0.049 & -0.049031 & 0.00003 \\
\hline$(0.15,0.10)$ & 0.003 & 0.00300832 & $8.32 \times 10^{-6}$ & -0.0955 & -0.0955913 & 0.00009 \\
\hline$(0.35,0.20)$ & 0.028 & 0.0280282 & 0.000028 & -0.151 & -0.151122 & 0.00012 \\
\hline$(0.49,0.31)$ & 0.094178 & 0.0942695 & 0.00009 & -0.161138 & -0.161259 & 0.00012 \\
\hline$(0.57,0.45)$ & 0.23085 & 0.231107 & 0.00025 & -0.15759 & -0.157779 & 0.00018 \\
\hline$(0.69,0.52)$ & 0.373152 & 0.37359 & 0.00043 & -0.024856 & -0.0247779 & 0.00007 \\
\hline$(0.73,0.71)$ & 0.735986 & 0.737218 & 0.0012 & 0.046718 & 0.0471139 & 0.00039 \\
\hline$(0.81,0.73)$ & 0.863298 & 0.864699 & 0.0014 & 0.227906 & 0.228805 & 0.00089 \\
\hline$(0.99,0.99)$ & 1.9406 & 1.94427 & 0.0036 & 0.950598 & 0.954343 & 0.0037 \\
\hline
\end{tabular}

TABLE 7: Comparison of results for $u_{x x}(n=2, m=25)$.

\begin{tabular}{lccccc}
\hline$\left(x_{i}, t_{i}\right)$ & $u_{x x}$ & $\left(u_{2}^{25}\right)_{x x}$ & $\left(e_{2}^{25}\right)_{x x}$ & $u_{t t}$ & $\left(u_{2}^{25}\right)_{t t}$ \\
\hline$(0.00,0.01)$ & 0.0002 & 0.000196032 & $3.96 \times 10^{-6}$ & -1.0000 & -1.00022 \\
$(0.10,0.05)$ & 0.005 & 0.00504296 & 0.00004 & -0.98 & -0.981028 \\
$(0.15,0.10)$ & 0.02 & 0.0201771 & 0.0001 & -0.955 & -0.956444 \\
$(0.35,0.20)$ & 0.08 & 0.0799329 & 0.00006 & -0.755 & 0.0002 \\
$(0.49,0.31)$ & 0.1922 & 0.191932 & 0.00026 & -0.5198 & -0.00102 \\
$(0.57,0.45)$ & 0.405 & 0.404939 & 0.00006 & -0.3502 & -0.351163 \\
$(0.69,0.52)$ & 0.5408 & 0.541206 & 0.0004 & -0.0478 & -0.0473431 \\
$(0.73,0.71)$ & 1.0082 & 1.00936 & 0.0011 & 0.0658 & 0.0014 \\
$(0.81,0.73)$ & 1.0658 & 1.06519 & 0.0006 & 0.3122 & 0.0670897 \\
$(0.99,0.99)$ & 1.9406 & 1.94427 & 0.0036 & 0.950598 & 0.0014 \\
\hline
\end{tabular}

TABLE 8: Comparison of results for $u_{x t}(n=2, m=25)$.

\begin{tabular}{lccc}
\hline$\left(x_{i}, t_{i}\right)$ & $u_{x t}$ & $\left(u_{2}^{25}\right)_{x t}$ & $\left(e_{2}^{25}\right)_{x t}$ \\
\hline$(0.00,0.01)$ & 0.000 & 0.000 & 0.000 \\
$(0.10,0.05)$ & 0.02 & 0.0199786 & 0.00002 \\
$(0.15,0.10)$ & 0.06 & 0.0602084 & 0.0002 \\
$(0.35,0.20)$ & 0.28 & 0.280396 & 0.00039 \\
$(0.49,0.31)$ & 0.6076 & 0.608402 & 0.0008 \\
$(0.57,0.45)$ & 1.026 & 1.02799 & 0.0019 \\
$(0.69,0.52)$ & 1.4352 & 1.43788 & 0.002 \\
$(0.73,0.71)$ & 2.0732 & 2.07878 & 0.005 \\
$(0.81,0.73)$ & 2.3652 & 2.37112 & 0.005 \\
$(0.99,0.99)$ & 3.9204 & 3.93184 & 0.011 \\
\hline
\end{tabular}

\section{Conflict of Interests}

The authors declare that there is no conflict of interests regarding the publication of this paper.

\section{References}

[1] N. Aronszajn, “Theory of reproducing kernels," Transactions of the American Mathematical Society, vol. 68, pp. 337-404, 1950.

[2] A. Berlinet and C. Thomas-Agnan, Reproducing Kernel Hilbert Space in Probability and Statistics, Kluwer Academic, Boston, Mass, USA, 2004.

[3] M. Cui and Y. Lin, Nonlinear Numercial Analysis in the Reproducing Kernel Space, Nova Science, New York, NY, USA, 2008. 
[4] A. Daniel, Reproducing Kernel Spaces and Applications, vol. 143 of Operator Theory: Advances and Applications, Springer, New York, NY, USA, 2003.

[5] C. Minggen and C. Zhong, "How to solve nonlinear operator equation $A\left(v^{2}\right)+C v=f$," Applied Mathematics and Computation, vol. 153, no. 2, pp. 403-416, 2004.

[6] M. Cui and C. Li, "How to solve the equation $\mathrm{AuBu}+\mathrm{Cu}=$ f," Applied Mathematics and Computation, vol. 133, no. 2-3, pp. 643-653, 2002.

[7] M. Cui and F. Geng, "Solving singular two-point boundary value problem in reproducing kernel space," Journal of Computational and Applied Mathematics, vol. 205, no. 1, pp. 6-15, 2007.

[8] C.-1. Li and M.-g. Cui, "The exact solution for solving a class nonlinear operator equations in the reproducing kernel space," Applied Mathematics and Computation, vol. 143, no. 2-3, pp. 393-399, 2003.

[9] M. Cui and Y. Lin, Nonlinear Numerical Analysis in the Reproducing Kernel Space, Nova Science Publishers, New York, NY, USA, 2008.

[10] H. Du and J. Shen, "Reproducing kernel method of solving singular integral equation with cosecant kernel," Journal of Mathematical Analysis and Applications, vol. 348, no. 1, pp. 308314, 2008.

[11] F. Geng, "A new reproducing kernel Hilbert space method for solving nonlinear fourth-order boundary value problems," Applied Mathematics and Computation, vol. 213, no. 1, pp. 163$169,2009$.

[12] F. Geng, "Solving singular second order three-point boundary value problems using reproducing kernel Hilbert space method," Applied Mathematics and Computation, vol. 215, no. 6, pp. 2095-2102, 2009.

[13] F. Geng and M. Cui, "Solving singular nonlinear two-point boundary value problems in the reproducing kernel space," Journal of the Korean Mathematical Society, vol. 45, no. 3, pp. 631-644, 2008.

[14] F. Geng and M. Cui, "Solving a nonlinear system of second order boundary value problems," Journal of Mathematical Analysis and Applications, vol. 327, no. 2, pp. 1167-1181, 2007.

[15] F. Geng and M. Cui, "Solving singular nonlinear second-order periodic boundary value problems in the reproducing kernel space," Applied Mathematics and Computation, vol. 192, no. 2, pp. 389-398, 2007.

[16] Y. Li, F. Geng, and M. Cui, “The analytical solution of a system of nonlinear differential equations," International Journal of Mathematical Analysis, vol. 1, no. 9-12, pp. 451-462, 2007.

[17] J. Li, "A computational method for solving singularly perturbed two-point singular boundary value problem," International Journal of Mathematical Analysis, vol. 2, no. 21-24, pp. 10891096, 2008.

[18] Y. Lin, P. Chung, and M. Cui, "A solution of an infinite system of quadratic equations in reproducing kernel space," Complex Analysis and Operator Theory, vol. 1, no. 4, pp. 571-579, 2007.

[19] Y. Zhou, M. Cui, and Y. Lin, "Numerical algorithm for parabolic problems with non-classical conditions," Journal of Computational and Applied Mathematics, vol. 230, no. 2, pp. 770-780, 2009.

[20] Y.-1. Wang and L. Chao, "Using reproducing kernel for solving a class of partial differential equation with variable-coefficients," Applied Mathematics and Mechanics. English Edition, vol. 29, no. 1, pp. 129-137, 2008.
[21] H. Yao and Y. Lin, "New algorithm for solving a nonlinear hyperbolic telegraph equation with an integral condition," International Journal for Numerical Methods in Biomedical Engineering, vol. 27, no. 10, pp. 1558-1568, 2011.

[22] A. Guezane-Lakoud, N. Bendjazia, and R. Khaldi, "Galerkin method applied to telegraph integro-differential equation with a weighted integral condition," Boundary Value Problems, vol. 2013, article 102, 2013.

[23] B. Basirat and M. A. Shahdadi, "Solution of nonlinear integrodifferential equations with initial conditions by bernstein operational matrix of derivative," International Journal of Modern Nonlinear Theory and Application, vol. 2, pp. 141-149, 2013.

[24] Z. P. Atabakan, A. K. Nasab, A. Kılıçman, and Z. K. Eshkuvatov, "Numerical solution of nonlinear Fredholm integro-differential equations using spectral homotopy analysis method," Mathematical Problems in Engineering, vol. 2013, Article ID 674364, 9 pages, 2013.

[25] L. Yang and M. Cui, "New algorithm for a class of nonlinear integro-differential equations in the reproducing kernel space," Applied Mathematics and Computation, vol. 174, no. 2, pp. 942960, 2006.

[26] M. Cui and H. Du, "Representation of exact solution for the nonlinear Volterra-Fredholm integral equations," Applied Mathematics and Computation, vol. 182, no. 2, pp. 1795-1802, 2006.

[27] O. Abu Arqub, M. Al-Smadi, and S. Momani, "Application of reproducing kernel method for solving nonlinear FredholmVolterra integrodifferential equations," Abstract and Applied Analysis, vol. 2012, Article ID 839836, 16 pages, 2012.

[28] M. Al-Smadi, O. Abu Arqub, and N. Shawagfeh, "Approximate solution of BVPs for 4th-order IDEs by using RKHS method," Applied Mathematical Sciences, vol. 6, no. 49-52, pp. 2453-2464, 2012. 


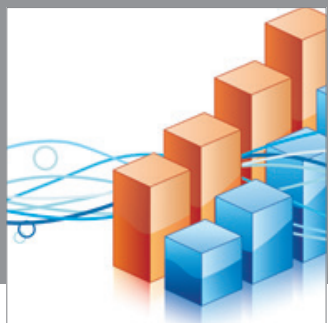

Advances in

Operations Research

mansans

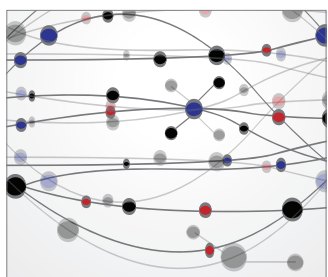

The Scientific World Journal
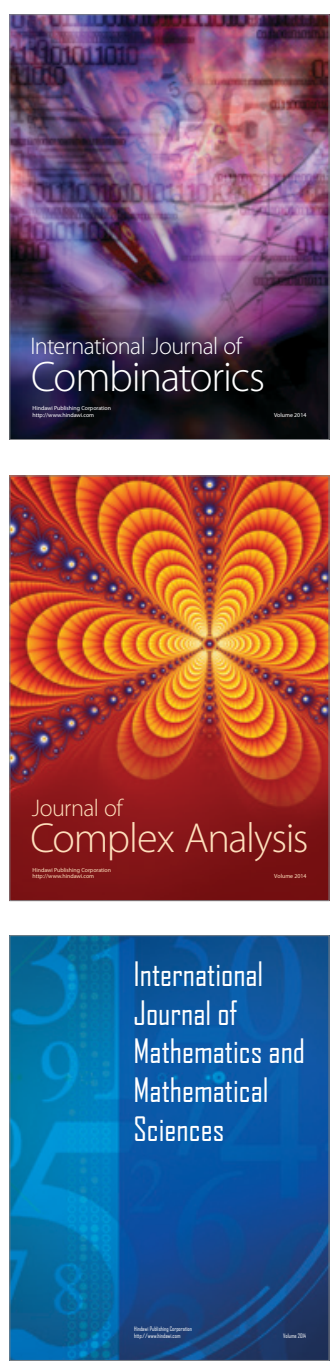
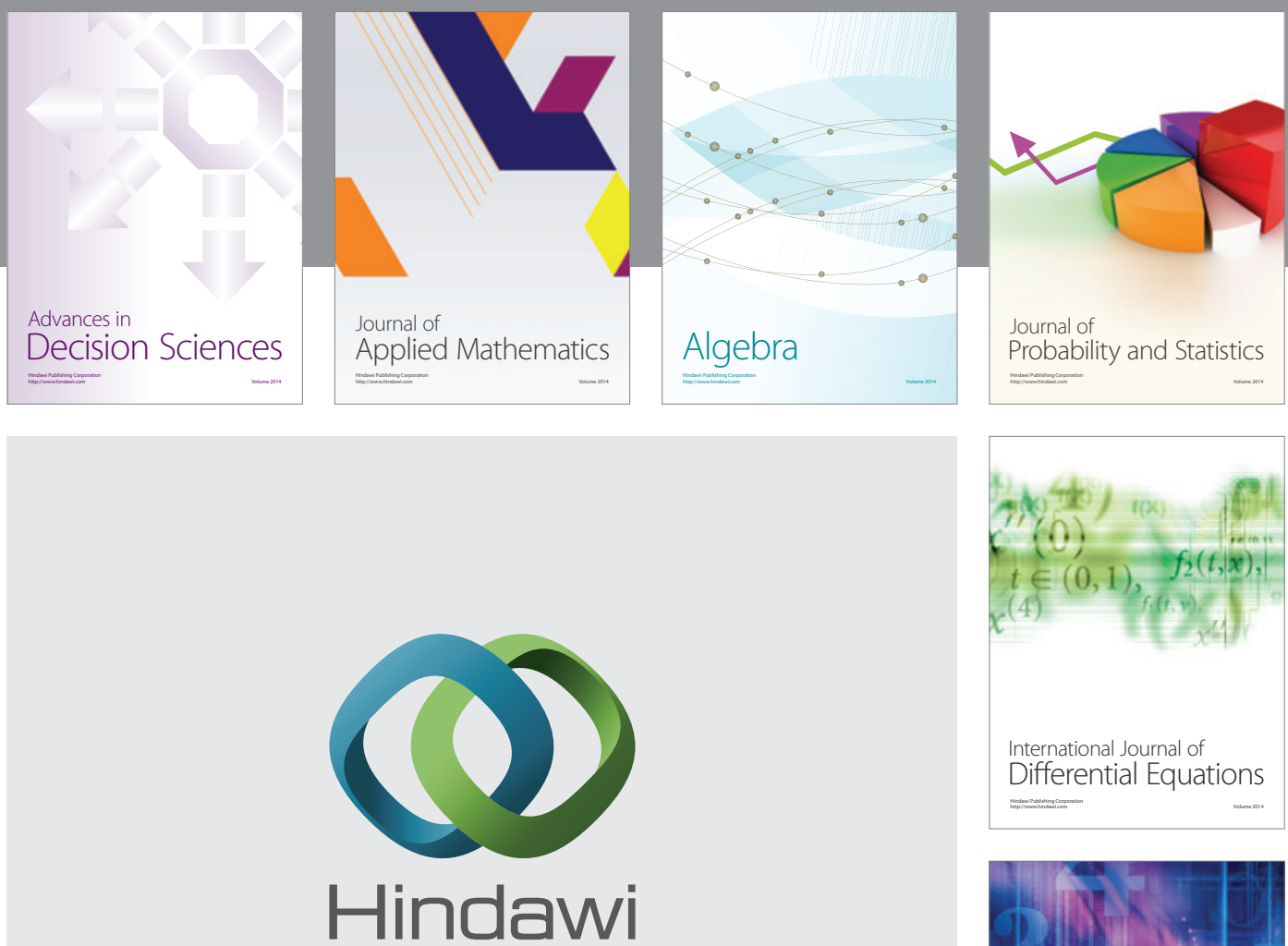

Submit your manuscripts at http://www.hindawi.com
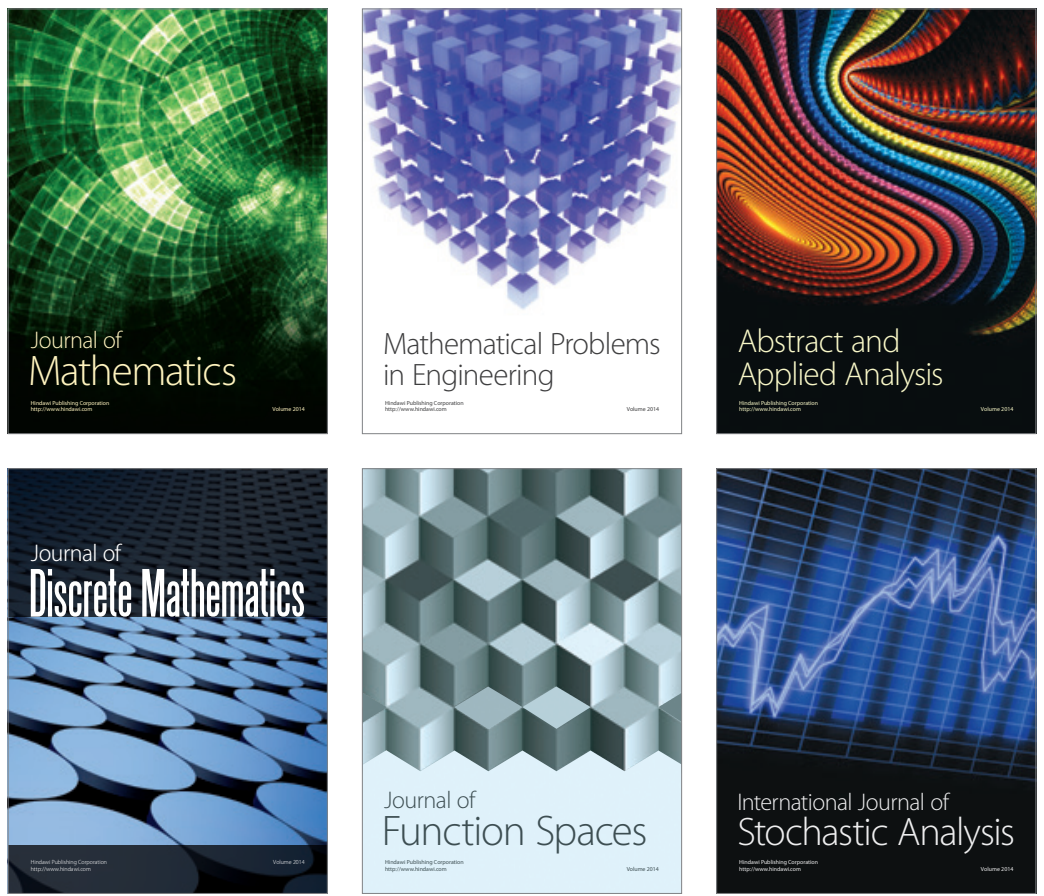

Journal of

Function Spaces

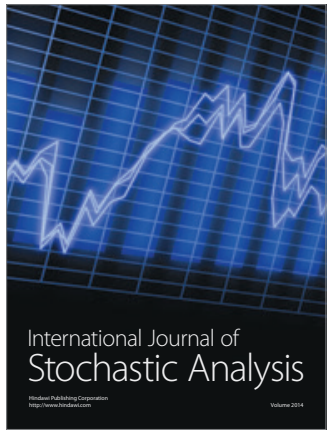

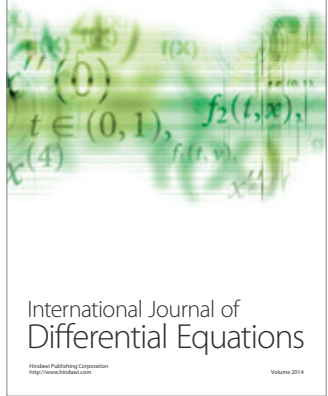
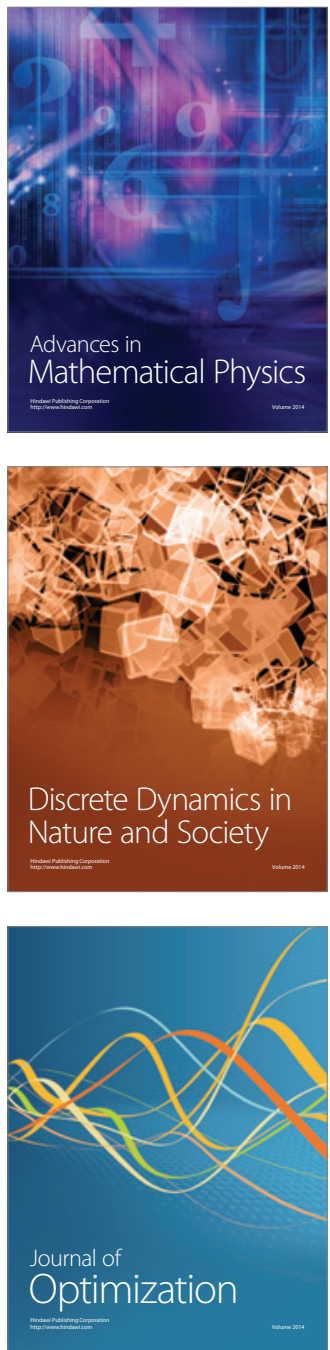